\title{
Knockdown of LKB1 Sensitizes Endometrial Cancer Cells via AMPK Activation
}

\author{
Seung Bae Rho ${ }^{1}$, Hyun Jung Byun ${ }^{2}$, Boh-Ram Kim ${ }^{2, *}$ and Chang Hoon Lee ${ }^{2, *}$ \\ ${ }^{1}$ Division of Translational Science, Research Institute, National Cancer Center, Goyang 10408, \\ ${ }^{2}$ BK21 FOUR Team and Integrated Research Institute for Drug Development, College of Pharmacy, Dongguk University, Seoul \\ 10326, Republic of Korea
}

\begin{abstract}
Metformin is an anti-diabetic drug and has anticancer effects on various cancers. Several studies have suggested that metformin reduces cell proliferation and stimulates cell-cycle arrest and apoptosis. However, the definitive molecular mechanism of metformin in the pathophysiological signaling in endometrial tumorigenesis and metastasis is not clearly understood. In this study, we examined the effects of metformin on the cell viability and apoptosis of human cervical HeLa and endometrial HEC-1-A and KLE cancer cells. Metformin suppressed cell growth in a dose-dependent manner and dramatically evoked apoptosis in HeLa cervical cancer cells, while apoptotic cell death and growth inhibition were not observed in endometrial (HEC-1-A, KLE) cell lines. Accordingly, the p27 and p21 promoter activities were enhanced while Bcl-2 and IL-6 activities were significantly reduced by metformin treatment. Metformin diminished the phosphorylation of mTOR, p70S6K and 4E-BP1 by accelerating adenosine monophosphateactivated kinase (AMPK) in HeLa cancer cells, but it did not affect other cell lines. To determine why the anti-proliferative effects are observed only in HeLa cells, we examined the expression level of liver kinase B1 (LKB1) since metformin and LKB1 share the same signalling system, and we found that the LKB1 gene is not expressed only in HeLa cancer cells. Consistently, the overexpression of LKB1 in HeLa cancer cells prevented metformin-triggered apoptosis while LKB1 knockdown significantly increased apoptosis in HEC-1-A and KLE cancer cells. Taken together, these findings indicate an underlying biological/physiological molecular function specifically for metformin-triggered apoptosis dependent on the presence of the $L K B 1$ gene in tumorigenesis.
\end{abstract}

Key Words: Metformin, LKB1, AMPK, HeLa, Endometrial cancer, Apoptosis

\section{INTRODUCTION}

Metformin (1,1-dimethylbiguanide hydrochloride) is generally an inexpensive and well-tolerated member of the biguanide class (Triggle and Ding, 2017). Around the world, it is the most widely prescribed anti-diabetic drug, and it also helps prevent cardiovascular disease and suppress metabolic syndrome (Evans et al., 2005; Ben Sahra et al., 2010). Various preclinical studies have indicated that metformin can suppress cell proliferation, trigger apoptotic cell death, provoke cell cycle arrest in vitro, and diminish the occurrence and growth of tumors in an in vivo orthotopic or xenograft mouse model system (Buzzai et al., 2007; Ben Sahra et al., 2008; Zhao et al., 2015; Zi et al., 2015). Metformin can be used as a sensitizer or can be combined with standard chemo-therapeutic agents and radiotherapy to treat tumors (Fasih et al., 2014; Qu et al.,
2014; Zhang et al., 2014; Lengyel et al., 2015; Uehara et al., 2015; Zhu et al., 2021). Furthermore, metformin plays an important role in targeting tumor stem cells and in reverting the epithelial-mesenchymal transition, a key process in tumor metastasis (Barriere et al., 2013; Nangia-Makker et al., 2014; Lei et al., 2017). Thus, metformin has been recognized as a potential anticancer agent. However, there is a need to conduct further studies on why patients show differences in response to metformin and with the anticancer action and mechanism of metformin.

AMPK is an enzyme that plays an important role in cellular energy homeostasis, largely regulating glycolysis and fatty acid oxidation, and it is also associated with cell growth and proliferation in eukaryotes (Steinberg and Kemp, 2009; Hardie, 2011; Yuan et al., 2013; Garcia and Shaw, 2017). Functionally, AMPK is accelerated by the AMP:ATP ratio contributed by the

\section{Open Access https://doi.org/10.4062/biomolther.2021.131}

This is an Open Access article distributed under the terms of the Creative Commons Attribution Non-Commercial License (http://creativecommons.org/licenses/by-nc/4.0/) which permits unrestricted non-commercial use, distribution, and reproduction in any medium, provided the original work is properly cited.
Received Aug 7, 2021 Revised Aug 31, 2021 Accepted Sep 7, 2021 Published Online Oct 5, 2021

\section{*Corresponding Authors}

E-mail: qtrami@gmail.com (Kim BR), uatheone@dongguk.edu (Lee CH) Tel: +82-31-961-5213 (Kim BR), +82-31-961-5213 (Lee CH) Fax: +82-31-961-5206 (Kim BR), +82-31-961-5206 (Lee CH) 
metabolic conditions, including obstructing ATP production due to deficiency of oxygen or glucose. The acceleration in response to the promotion of AMP involves phosphorylation by an up-stream target kinase, the liver kinase B1 (LKB1) (Towler and Hardie, 2007).

The LKB1 protein is the main up-stream kinase of AMPK, implying that the inhibitory effects of LKB1 on cancer can be controlled by AMPK (Shaw et al., 2004; Li et al., 2017). In cancer, AMPK can be a promising target to treat tumors. Tumor cells have characteristic metabolic transformations from the metabolic state of the healthy original cell, and as a major metabolic modulator, AMPK can regulate metastasis. In addition, AMPK can act to suppress tumorigenesis by controlling the cell proliferation, cellular stress responses, cell growth, autophagy, and cell polarity (Wang and Guan, 2009). Therefore, the possible anti-tumorigenic function of metformin can be regulated by its role in accelerating AMPK, which in turn suppresses the phosphorylation of a mammalian target of rapamycin (mTOR) (Li et al., 2017; Mallik and Chowdhury, 2018). Considering the defined role of AMPK in tumorigenesis, the goal of our research is to elucidate the potential biological effects of metformin on the cervical and endometrial cancers, as well as demonstrate the underlying molecular function.

In this manuscript, we found that metformin showed different inhibitory effects on the growth of HeLa (low LKB1 expression), HEC-1-A and KLE cells (high LKB1 expression). We also found that gene silencing of LKB1 expression recovers the metformin-induced growth inhibition in HEC-1-A and KLE cells.

\section{MATERIALS AND METHODS}

\section{Cell lines, reagents, and antibodies}

Human cervical carcinoma cell lines HeLa, endometrial cancer cell lines (HEC-1-A and KLE) and human embryonic kidney 293T (HEK293T) cells were obtained from the American Type Culture Collection (ATCC, Manassas, VA, USA) and were grown in monolayer cultures in accordance with ATCC recommendations. Metformin was purchased from Sigma (St. Louis, MO, USA). The following antibodies used in this study were anti-caspase-3, anti-LKB1, anti-Bcl-2, anti-Bcl-xL, antiBax, anti-AMPK, anti-phospho-AMPK, anti-mTOR, anti-phospho-mTOR (Ser2448), anti-p70S6K, anti-phospho-p70S6K (Thr421), anti-4E-BP1, anti-phospho-4E-BP1 (Thr70) (Cell Signaling, Beverly, MA, USA), anti-PARP (BD Biosciences, San Jose, CA, USA), anti-GAPDH, anti-cyclin D1, anti-CDK4, anti-p27, anti-p16, anti-IL-6 (Santa Cruz Biotechnology, Santa Cruz, CA, USA), anti-p21 (Ab-1; Oncogene, Cambridge, MA, USA), and anti- $\beta$-actin (Sigma).

\section{Cell proliferation assay and flow cytometric analysis of apoptosis}

Cell viability was evaluated using the CellTiter-Glo luminescent assay kit (Promega, Madison, WI, USA), according to the supplier's instructions. Briefly, cells were grown at a density of $4.6 \times 10^{3}$ per well in 96-well plates. After $24 \mathrm{~h}$, the cells were transfected with various concentrations of metformin. Cell viability was assessed using the CellTiter-Glo reagent (Promega) according to the manufacturer's instructions.

Apoptosis of HeLa, HEC-1-A, and KLE cells was analyzed utilizing flow cytometry as previously reported (Byun et al.,
2012). In brief, $2.8 \times 10^{5}-3.2 \times 10^{5}$ cells/well were seeded in 60 $\mathrm{mm}$ plates. Whole cells were incubated with FITC-labeled Annexin $\mathrm{V}$ and propidium iodide $(\mathrm{PI})$ for $15 \mathrm{~min}$ in accordance with supplier's instructions (BD Pharmingen, Mississauga, ON, Canada) and were developed utilizing fluorescence activated cell sorting (FACS) Vantage BD FACSCalibur flow cytometer (Becton-Dickinson, Franklin Lakes, NJ, USA).

\section{Assay of caspase-3 and PARP activity, construction of small interfering RNA (siRNA) for LKB1 gene}

The activity of caspase-3 and PARP in HeLa, HEC-1-A, and KLE cancer cells were developed as previously reported (Zhang and Snyder, 1992; Kang et al., 2012). Various siRNA sequences from the LKB1 RNA sequence were prepared utilizing a siRNA Construction kit (Ambion, Austin, TX, USA). After selection of two clones, the prepared sequences were then transfected with oligofectamine (Thermo Fisher Scientific, Carlsbad, CA, USA) in accordance with supplier's recommendation. TRIZOL Reagent (Thermo Fisher Scientific) was used to isolate whole RNA and reverse transcription (RT) PCR was then conducted. LKB1 expression was measured via western blot analysis.

\section{Western blot analysis}

Western blot analysis was conducted in accordance with a previous report (Kang et al., 2020). Briefly, whole cells were lysed in standard RIPA lysis buffer in ice for $30 \mathrm{~min}$. After centrifugation, the protein concentration was evaluated utilizing the Bradford assay. About $15-20 \mu \mathrm{g}$ protein extracts were separated by $10-12 \%$ SDS-PAGE and were transferred onto a nitrocellulose membrane (BioRad, CA, USA). After blocking, the membranes were probed for caspase-3, PARP, $\beta$-actin, p21, p27, p16, cyclin D1, CDK4, IL-6, Bcl-2, Bcl-xL, Bax, AMPK, phospho-AMPK, mTOR, phospho-mTOR, p70S6K, phosphop70S6K, 4E-BP1, and phospho-4E-BP1, respectively. The protein bands were visualized utilizing an ECL chemoluminescence detection kit (GE Healthcare, Piscataway, NJ, USA).

\section{Luciferase activity assay}

In vitro luciferase activity was assessed using a dual luciferase reporter assay system (Promega). Cells were introduced with plasmids containing p27-Luc, p21-Luc, Bcl-2-Luc, and IL-6-Luc, in which the promoter of p27, p21, Bcl-2, and IL-6 control the expression of luciferase, respectively (Kang et al., 2012; Rho et al., 2012). In brief, cells were seeded to $85 \%$ confluence and co-transfected with the promoter-luciferase plasmids containing the Renilla luciferase reporter for $24 \mathrm{~h}$. Following lysis with the radio-immunoprecipitation buffer, the lysates were cleared by centrifugation at $12,000 \mathrm{rpm}$ for 20 $\mathrm{min}$, and the cell extracts were incubated with the luciferase substrate reagent for $30 \mathrm{~min}$ at room temperature according to the supplier's instructions. Then, a $5 \mu \mathrm{L}$ aliquot of each sample was quantified using a MicroLumat Plus LB96V luminometer (Berthold Technologies, Bad Wildbad, Germany). The ratio was normalized for Renilla luciferase activity to correct for variation in transfection efficiency.

\section{Statistical analysis}

All the data are expressed as the mean \pm SD and were estimated using Student's $t$-test and analyses of variance according to the number of groups compared. Significant differences $(p<0.05)$ are depicted with asterisks in each figure. The analy- 
A
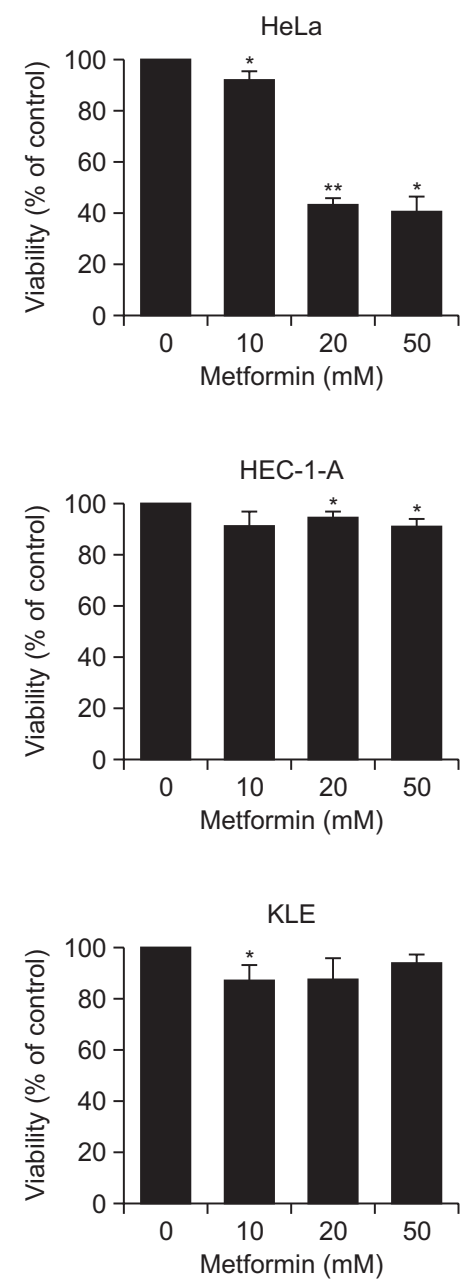

HeLa
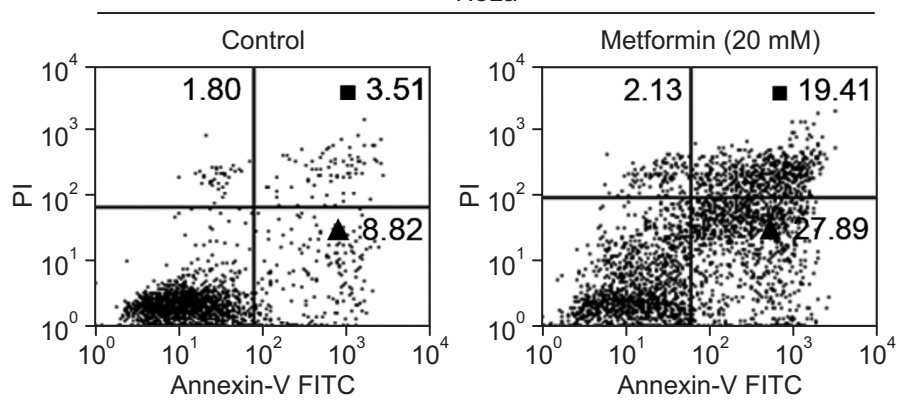

HEC-1-A
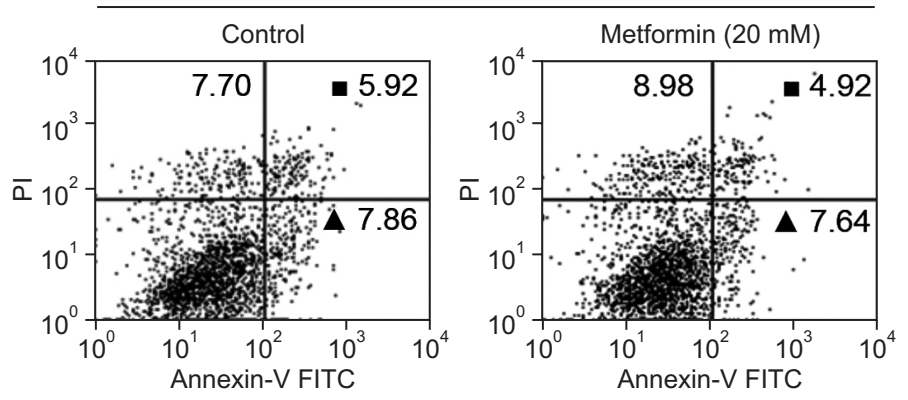

KLE
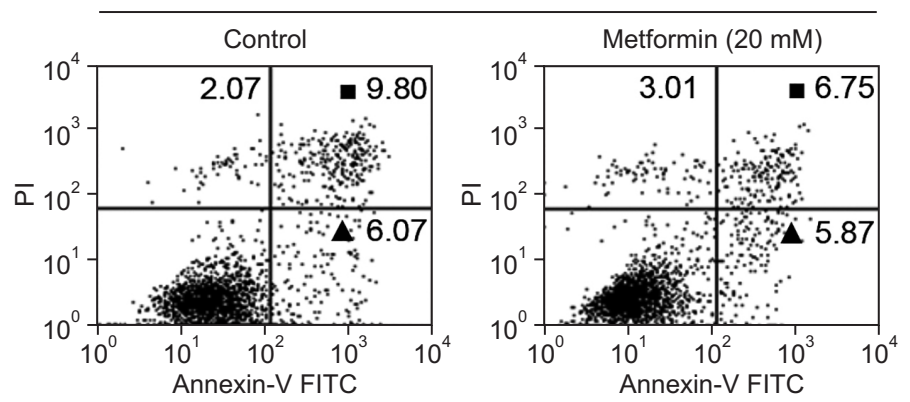

B
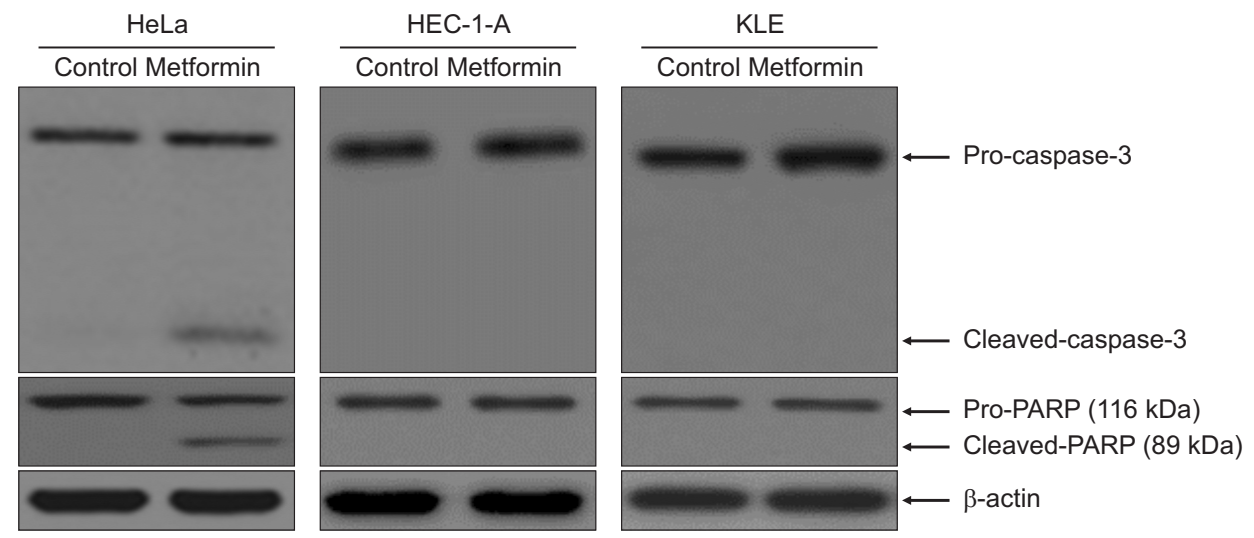

Fig. 1. Metformin selectively activates apoptotic cell death in human cervical and endometrial cancer cell lines. (A, B) Cell proliferation assay (A, left panel), FACS analysis (A, right panel), and caspase-3 and PARP assay (B). HeLa, HEC-1-A and KLE cells were treated with 0 , 10,20 and $50 \mathrm{mM}$ metformin, respectively. Relative values of cell proliferation were calculated as described in the supplier's protocols employing CellTiter-Glo assay system. Results represent the mean \pm SD of at least three independent experiments conducted in triplicate. ${ }^{*} p<0.05 ;{ }^{* *} p<0.01$ compared with the control $(0 \mathrm{mM})$. Subsequently, early- $(\boldsymbol{\Delta})$ and late-stage $(\boldsymbol{\square})$ apoptotic cell death triggers by metformin was performed with fluorescein isothiocyanate (FITC)-labeled Annexin V assay. All experiments were repeated at least three times with similar results. And then, caspase-3 and PARP cleavages induced by metformin treatment. Soluble protein extracts were detected by western blot analysis for cleaved caspase-3 and cleaved PARP. $\beta$-actin served as a control. All results seen are representative of three independent experiments. 
A

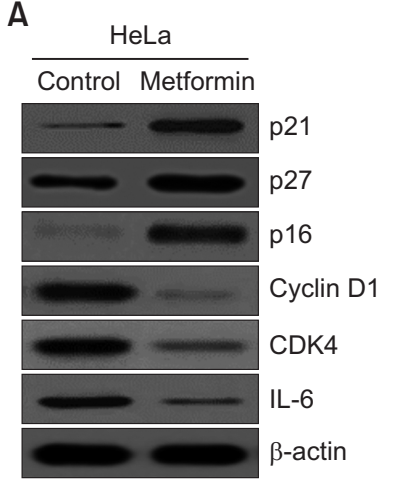

B

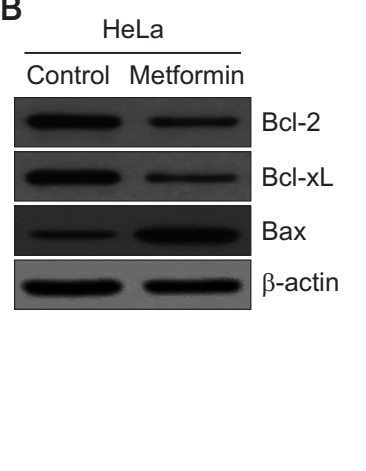

C

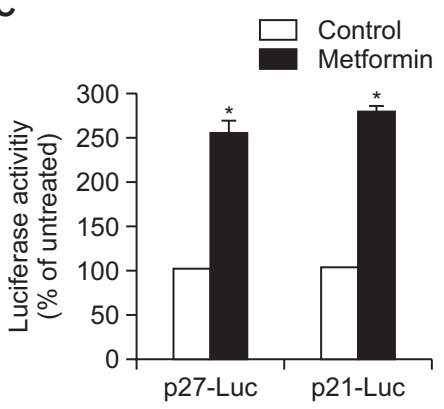

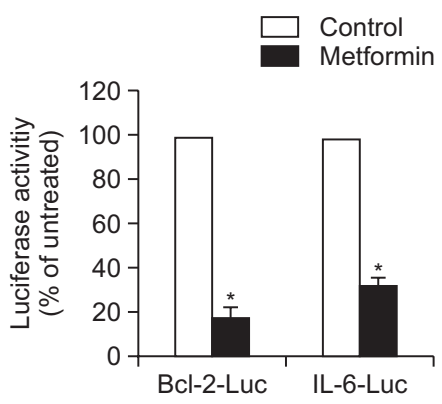

Fig. 2. Metformin controls the expression level of cell cycle- and apoptosis-associated proteins. (A, B) HeLa cells were treated with metformin. Cells were harvested and added with lysis buffer. Total cell lysates were used western blot analysis. Expression levels of cell cycle and apoptosis-related protein were visualized by indicated specific antibodies. All results were conducted at least three times with consistent and similar results. (C) The activity of metformin on the p27, p21, Bcl-2 and IL-6 transcription using an each p27, p21, Bcl-2 and IL-6 promoter construct fused to the luciferase gene in human HeLa cells. The luciferase activity was measured with a luminometer. Results show the mean \pm SD of at least three independent experiments performed in triplicate. ${ }^{*} p<0.05$ versus the control group.

ses were performed using the SPSS 20 software (Statistical Product and Service Solutions, Chicago, IL, USA).

\section{RESULTS}

\section{Metformin selectively suppresses cell growth in HeLa cells but not HEC-1-A, and KLE cells}

To elucidate the effects that metformin had in inhibiting growth of both human cervical HeLa and endometrial (HEC1-A, KLE) cancer cell lines, the cells were treated with various concentrations, and the cell viability and apoptotic cell death were respectively observed using the CellTiter-Glo assay and flow cytometry, respectively. As shown in Fig. 1, metformin gradually inhibited cell proliferation in a dose-dependent manner and it significantly accelerated apoptosis in HeLa cells, while apoptotic cell death and growth suppression were not observed in endometrial (HEC-1-A, KLE) cell lines (Fig. 1A). In addition, cleaved caspase-3 and PARP activities were enhanced in the metformin-treated HeLa cells, which was essential to stimulate apoptosis (Fig. 1B). These findings strongly indicate that metformin can be a cell-specific trigger to inhibit HeLa cervical cancer cell growth.

\section{Metformin regulates the expression level of cell cycle- and apoptosis-associated proteins}

The underlying molecular mechanism by which metformin played its role in attenuating HeLa cervical cell proliferation is explored by conducting a Western blot analysis to demonstrate the important signals related to the cell cycle and apoptosis function. As presented in Fig. 2A, treatment with metformin considerably suppressed the expression of cyclin D1 (CDK1), CDK4, and IL-6, but it accelerated the expression of p16 and p21 or p27. Next, we investigated metformin-treated cell growth by observing the expression of apoptosis-associated proteins $\mathrm{Bcl}-2, \mathrm{Bcl}-\mathrm{xL}$, and $\mathrm{Bax}$. These proteins are wellknown essential regulators involved in cell growth and apoptosis. As seen in Fig. 2B, Bcl-2 and Bcl-xL expression were significantly diminished, whereas Bax was considerably accelerated by metformin treatment, compared with the control. Furthermore, the luciferase reporter-gene assay validated that metformin dramatically accelerated the transcription levels of p27 and p21, while Bcl-2 and IL-6 promoter activities were remarkably diminished (Fig. 2C). Collectively, these results possibly indicate that metformin can be used as an anti-tumor drug in cervical cancer therapy.

\section{LKB1 overexpression desensitizes HeLa cells to $20 \mathrm{mM}$ metformin}

To investigate why $20 \mathrm{mM}$ metformin specifically inhibits the proliferation of HeLa cells, we examined the differences in expression of LKB1 in HeLa, HEC-1-A, and KLE cells by a reverse transcriptase-polymerase chain reaction (RT-PCR) and western blotting. We found that the LKB1 is not expressed only in HeLa cancer cells but expressed in HEC-1-A and KLE cells (Fig. 3A). LKB1 overexpression was confirmed in HeLa cells using plasmid containing LKB1 (LKB1) (Fig. 3B). Then, the effect of LKB1 overexpression on $20 \mathrm{mM}$ metformin-induced growth-inhibition of HeLa cells was examined using flow cytometry. The overexpression of LKB1 in HeLa cancer cells reduced $20 \mathrm{mM}$ metformin-induced apoptosis (Fig. 3C). After $20 \mathrm{mM}$ metformin treatment, cleaved caspase-3 and PARP levels were significantly higher, but the ectopic expression of LKB1 reduces metformin-induced cleavage of caspase-3 and PARP (Fig. 3D). These results indicate that a loss of LKB1 is essential for $20 \mathrm{mM}$ metformin-induced growth inhibition and apoptosis of HeLa cancer cells.

\section{A loss of LKB1 expression by LKB1 siRNA sensitizes HEC- 1-A and KLE cells}

Then, we examined the effects of LKB1 siRNA (siLKB1) on HEC-1-A and KLE cells, which expresses LKB1. Reduced expressions of LKB1 by siLKB1 in HEC-1-A, sensitized to $20 \mathrm{mM}$ metformin, leading to the increases in portions of apoptotic cells and the cleavage of caspase- 3 and PARP (Fig. 4A, 4B). Same trends were observed in KLE cells (Fig. 4C, 4D). LKB1 inhibition might reverse nonresponsive HEC-1-A, and KLE cancer cells to cells vulnerable to $20 \mathrm{mM}$ metformin treatment.

\section{Metformin enhances phosphorylation of AMPK in siLKB1- treated KLE cells}

We examined whether phosphorylation of AMPK was ob- 
A

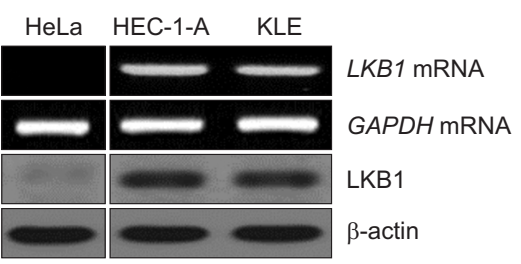

C

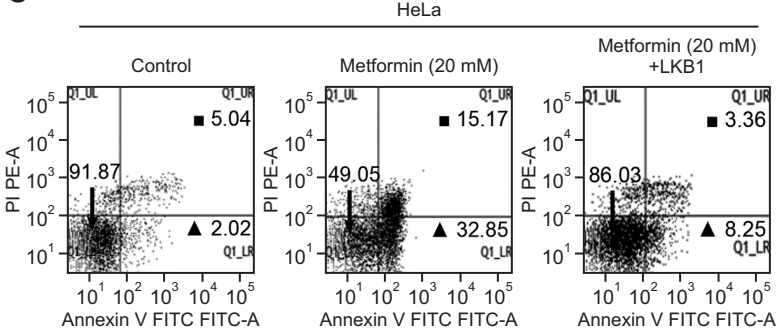

B

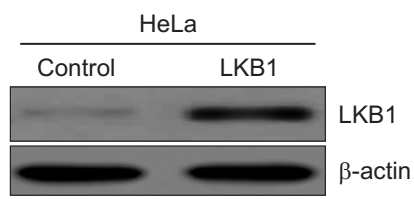

D

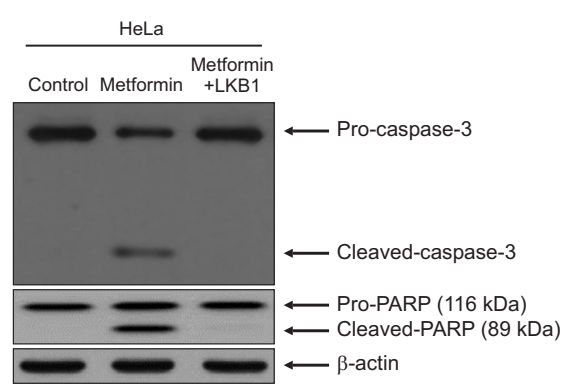

Fig. 3. Expression of $L K B 1$ gene in HeLa and endometrial cancer cell lines and effects of metformin on the LKB1-overexpressed HeLa cells. (A) Expression levels of $L K B 1$ mRNA and protein was analyzed by RT-PCR and western blot analysis. GAPDH and $\beta$-actin included as an mRNA or protein loading control, respectively. (B) LKB1 overexpression was confirmed by western blot analysis. (C) After treat/transfected with metformin or metformin plus $L K B 1$, flow cytometric analysis was conducted to calculate the early- ( $\mathbf{\Delta})$ and late- ( $\mathbf{\square})$ stage apoptotic death cells in HeLa cell lines. (D) Cells were collected and treated with lysis buffer. Total cell lysates were subsequently subjected to western blot analysis. Expression levels of cleaved caspase-3 and cleaved PARP protein were observed using indicated specific antibodies. All data were repeated at least three times with similar results.

A

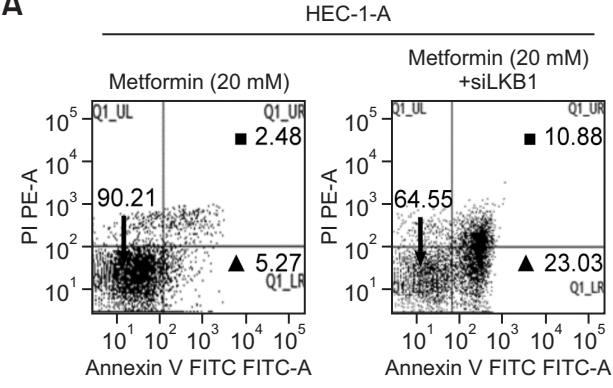

C

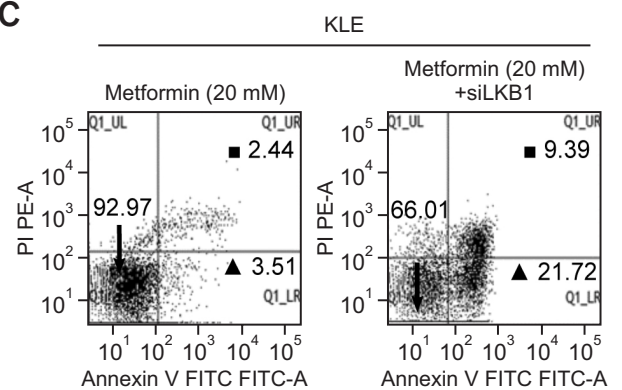

B

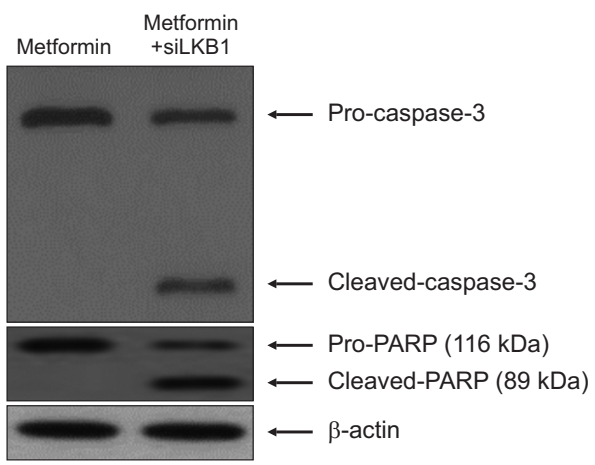

D

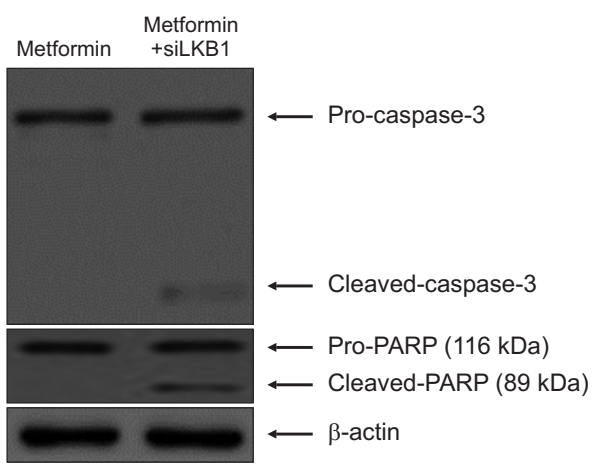

Fig. 4. siLKB1 sensitizes HEC-1-A and KLE cells. After treat/transfected with metformin or metformin plus siLKB1, flow cytometric analysis was conducted to calculate the early- $(\boldsymbol{\Delta})$ and late- $(\boldsymbol{\square})$ stage apoptotic cell death cells in HEC-1-A (A) and KLE cells (C). (B, D) Caspase-3 and PARP assay. HEC-1-A (B) and KLE cells (D) were collected and treated with lysis buffer. Total cell lysates were subsequently subjected to western blot analysis. Expression levels of cleaved caspase-3 and cleaved PARP protein were observed using indicated specific antibodies. All data were repeated at least three times with similar results. 
A

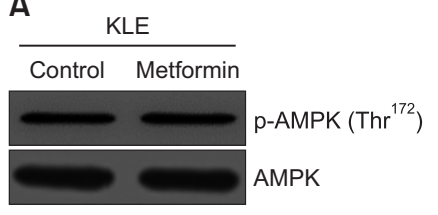

B

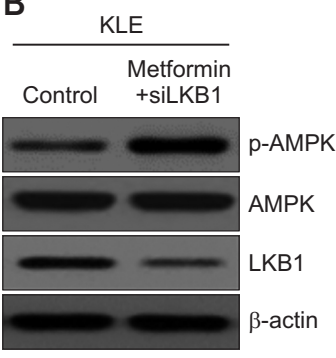

A

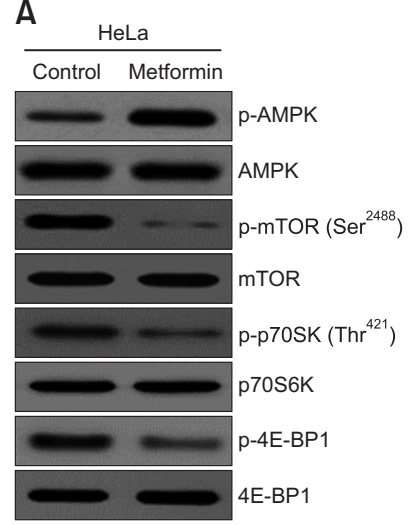

B

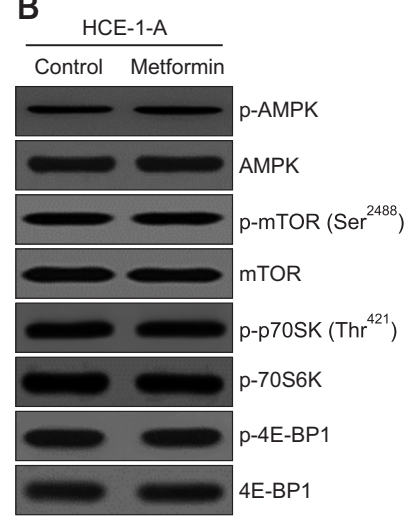

Fig. 5. Metformin enhances phosphorylation of Thr172 in AMPK in siLKB1-treated KLE endometrial cells. (A) Effects of metformin on phosphorylation of Thr172 in AMPK. (B) Metformin increases the phosphorylation of Thr172 in siLKB1-treated KLE cells. All experiments were carried out at least three times with consistent and similar results.

served in KLE cells insensitive to low-dose metformin. Accordingly, $20 \mathrm{mM}$ metformin did not cause AMPK phosphorylation in these cells (Fig. 5A). Interestingly, however, when LKB1 expression was lowered with siRNA and then $20 \mathrm{mM}$ metformin was treated, phosphorylation of AMPK was observed by 20 $\mathrm{mM}$ metformin (Fig. 5B). Therefore, these results suggested that the effect of metformin on phosphorylation of AMPK can be dependent on the loss of LKB1.

\section{Metformin inhibits the mTOR signaling pathway through AMPK activation in HeLa cells lacking LKB1 expression}

To elucidate the effects of metformin on AMPK signaling in low-dose metformin-sensitive HeLa cells and metformin-nonresponsive HEC-1-A tumor cells, we examined the phosphorylation of AMPK, mTOR, and mTOR down-stream targets such as p70S6K and 4E-BP1. Total cell lysates from the control HeLa cells and metformin-treated HeLa cells were examined through western blot analysis. As shown in Fig. 6A, metformin remarkably induced the activation of AMPK phosphorylation and reduced mTOR phosphorylation, as well as the phosphorylation of p70S6K and 4E-BP1 in HeLa cells (metformin sensitive). By contrast, no changes in the phosphorylation of AMPK, mTOR, p70S6K, and 4E-BP1 were observed in HEC1-A endometrial cancer cell (metformin nonresponsive) (Fig. $6 \mathrm{~B})$. Therefore, metformin might suppress the proliferation of LKB1-deficient HeLa cells through inhibition of the mTOR signaling pathway by activating AMPK.

\section{DISCUSSION}

Metformin is used as an oral anti-diabetic drug, and it is well-known to regulate cell growth and protein synthesis by suppressing the mTOR signaling cascade by accelerating ATM (ataxia telangiectasia mutated), LKB1, and then AMPK (Shaw et al., 2005; Towler and Hardie, 2007; Lee et al., 2019). According to the previously reported, the anti-cancer effects of metformin are generally exhibits on various human cancer types including breast (Zakikhani et al., 2006; Dowling et al., 2007), ovary (Algire et al., 2008; Gotlieb et al., 2008), lung (Algire et al., 2008; Brancher et al., 2021), colon (Buzzai et al., 2007) and prostate tumor cell lines (Ben Sahra et al., 2008).

LKB1 is inactivated approximately $30 \%$ through a somatic mutation in non-small cell lung cancer (NSCLC) cells and is

Fig. 6. Metformin can accelerate the phosphorylation of AMPK in HeLa cells (A) Phosphorylation of AMPK was confirmed in metformin-treated HeLa cells. (A) HeLa (without LKB1 gene) and (B) HEC-1-A (with LKB1) cells were treated with metformin, respectively. HEC-1-A cell lines compared to HeLa cervical cells. Then, whole cells were collected and added with lysis buffer. After harvest by centrifugation, equal amounts of protein $(20 \mu \mathrm{g})$ were separated on $8-12 \%$ SDS-PAGE, followed by western blot analysis with indicated specific antibodies (AMPK/phosphorylated AMPK, mTOR/phosphorylated mTOR, p70S6K/ phosphorylated p70S6K and 4E-BP1/phosphorylated 4E-BP1). Non-phosphorylated form (indicated AMPK, mTOR, p70S6K and 4E-BP1) included to verify equal loading of the samples, respectively. All data were conducted at least three times with consistent and similar results.

regarded as tumour suppressor (Liu et al., 2013). However, the biological and molecular mechanism dependent on LKB1 gene that provides the anti-cancer effect of metformin for endometrial cancers remain unclear.

Our data showed a growth inhibition effect of metformin on HeLa cells is consistent with the previous report (Fig. 1) (Xiao et al., 2012). Furthermore, metformin showed growth inhibition in HEC-1-A cells (Zhang et al., 2017) and invasion inhibitory effects in KLE cells (Suh et al., 2020), the growth inhibition effect of metformin in these cell lines was not observed in our data (Fig. 1).

AMPK/mTOR signaling cascade is usually under the control of LKB1. LKB1 is a major up-stream kinase responsible for AMPK phosphorylation. Loss of $L K B 1$ results in loss of AMPK signaling pathway. Therefore, loss of $L K B 1$ prevented the therapeutic effects of metformin, indicating that metformin treatment of mice accelerated AMPK activity in the liver and lowered blood glucose levels in an LKB1-dependent manner (Shaw et al., 2005; Li et al., 2018). However, in this study, we have discovered that LKB1 overexpression can specifically prevent metformin-induced growth inhibition and apoptotic cell death in HeLa cancer cells (Fig. 3C, 3D). Interestingly, siLKB1 can considerably induce apoptotic cell death in metformintreated HEC-1-A and KLE tumor cells (Fig. 4). Therefore, the results in Fig. 3 and 4 suggests that metformin-induced cell death via LKB1/AMPK axis is not valid in HeLa, HEC-1-A, and KLE tumor cells.

Interestingly, siLKB1 enhanced the phosphorylation of AMPK in metformin-treated KLE cells (Fig. 5B). In line with this observation, metformin enhanced the phosphorylation of AMPK also in HeLa cells deficient for LKB1 (Fig. 6A). These results clearly show that AMPK is activated by metformin even in the deficiency of LKB1. Kinases other than LKB1, namely 
CAMKK, ATM, and TAK1 have been reported to be able to activate AMPK (Sun et al., 2007; Herrero-Martin et al., 2009; Sanli et al., 2010; Gou et al., 2013; Fogarty et al., 2016; Loubiere et al., 2017; Chen et al., 2018).

LKB1 can act as a low energy sensor and acts as a negative regulator of apoptosis in normal cells (Shaw et al., 2004). Therefore, under the basic conditions, LKB1 acts as a sensor of low energy and maintains the ATP consumption process including protein synthesis through check via phosphorylation of TSC2 by AMPK (Shaw et al., 2004). In response to stress such as hypoglycemia, hypoxia, malnutrition, or mitochondrial toxins, LKB1 phosphorylates AMPK, blocking the ATP consumption process to offset the elevated AMP level and upregulating ATP production (Shaw et al., 2004). LKB1-induced activation of AMPK prevents normal cells from entering apoptosis in response to elevated AMP. However, in the case of cancer, metformin treatment suppressed mTOR activation and S6K phosphorylation in LKB1-deficient HeLa cells via TSC activity induced by AMPK phosphorylation (Fig. 6). Metformin induces cell death in HeLa and LKB1-knockdown KLE and HEC-1A cells presumably through this mechanism.

In general, since LKB1 is a tumor suppressor, LKB1 deficiency likely induces an increase in the oncogenic potential or growth of cancer cells (Shaw et al., 2004). Therefore, LKB1 deficient cells show high mitochondrial metabolism, as indicated by increased mitochondrial potential and oxygen-consumption rate (Whang et al., 2016). Accordingly, these cells appear to be vulnerable to substances that affect the mitochondrial function. Therefore, metformin treatment induces LKB1-independent activation of AMPK, thereby affecting mitochondria (Vial et al., 2019) and activating TSC through phosphorylation (Green et al., 2011), which seems to inhibit the activity of mTOR kinase.

In all, we propose loss of $\angle K B 1$ is involved in the anti-proliferative effects of metformin in HeLa cervical cancer cells. siLKB1 converts metformin non-responsive HEC-1-A and KLE cells to responsive to metformin. These results suggested that expression level of LKB1 might be a marker for responsiveness to metformin.

\section{CONFLICT OF INTEREST}

The authors declare no conflict of interest.

\section{ACKNOWLEDGMENTS}

This study was partially supported by a grant from the National Cancer Center (NCC-0810410-3), BK21 FOUR program, the Basic Science Research Program, through the National Research Foundation (NRF) of Korea (NRF2020R1A2C3004973, NRF-2018R1A5A2023127, NRF2020M3E5E2038356), and Global PhD. Fellowship through the NRF of Korea (NRF-2018H1A2A1061990).

We would like to thank Dr. Richard Yoo (University of Michigan at Ann Arbor, USA) for critically reading of the manuscript.

\section{REFERENCES}

Algire, C., Zakikhani, M., Blouin, M. J., Shuai, J. H. and Pollak, M.
(2008) Metformin attenuates the stimulatory effect of a high-energy diet on in vivo LLC1 carcinoma growth. Endocr. Relat. Cancer 15, 833-839.

Barriere, G., Tartary, M. and Rigaud, M. (2013) Metformin: a rising star to fight the epithelial mesenchymal transition in oncology. Anticancer Agents Med. Chem. 13, 333-340.

Ben Sahra, I., Laurent, K., Loubat, A., Giorgetti-Peraldi, S., Colosetti, P., Auberger, P., Tanti, J. F., Le Marchand-Brustel, Y. and Bost, F. (2008) The antidiabetic drug metformin exerts an antitumoral effect in vitro and in vivo through a decrease of cyclin D1 level. Oncogene 27, 3576-3586.

Ben Sahra, I., Le Marchand-Brustel, Y., Tanti, J. F. and Bost, F. (2010) Metformin in cancer therapy: a new perspective for an old antidiabetic drug? Mol. Cancer Ther. 9, 1092-1099.

Brancher, S., Støer, N. C., Weiderpass, E., Damhuis, R. A., Johannesen, T. B., Botteri, E. and Strand, T. E. (2021) Metformin use and lung cancer survival: a population-based study in Norway. Br. J. Cancer 124, 1018-1025.

Buzzai, M., Jones, R. G., Amaravadi, R. K., Lum, J. J., DeBerardinis, R. J., Zhao, F., Viollet, B. and Thompson, C. B. (2007) Systemic treatment with the antidiabetic drug metformin selectively impairs p53-deficient tumor cell growth. Cancer Res. 67, 6745-6752.

Byun, H. J., Kim, B. R., Yoo, R., Park, S. Y. and Rho, S. B. (2012) sMEK1 enhances gemcitabine anti-cancer activity through inhibition of phosphorylation of Akt/mTOR. Apoptosis 17, 1095-1103.

Chen, X., Li, K. and Zhao, G. (2018) propofol inhibits hela cells by impairing autophagic flux via AMP-activated protein kinase (AMPK) activation and endoplasmic reticulum stress regulated by calcium. Med. Sci. Monit. 24, 2339-2349.

Dowling, R. J., Zakikhani, M., Fantus, I. G., Pollak, M. and Sonenberg, N. (2007) Metformin inhibits mammalian target of rapamycindependent translation initiation in breast cancer cells. Cancer Res. 67, 10804-10812.

Evans, J. M., Donnelly, L. A., Emslie-Smith, A. M., Alessi, D. R. and Morris, A. D. (2005) Metformin and reduced risk of cancer in diabetic patients. BMJ 330, 1304-1305.

Fasih, A., Elbaz, H. A., Huttemann, M., Konski, A. A. and Zielske, S. P. (2014) Radiosensitization of pancreatic cancer cells by metformin through the AMPK pathway. Radiat. Res. 182, 50-59.

Fogarty, S., Ross, F. A., Vara Ciruelos, D., Gray, A., Gowans, G. J. and Hardie, D. G. (2016) AMPK causes cell cycle arrest in LKB1deficient cells via activation of CAMKK2. Mol. Cancer Res. 14, 683-695.

Garcia, D. and Shaw, R. J. (2017) AMPK: mechanisms of cellular energy sensing and restoration of metabolic balance. Mol. Cell 66, 789-800.

Gotlieb, W. H., Saumet, J., Beauchamp, M. C., Gu, J., Lau, S., Pollak, M. N. and Bruchim, I. (2008) In vitro metformin anti-neoplastic activity in epithelial ovarian cancer. Gynecol. Oncol. 110, 246-250.

Gou, S., Cui, P., Li, X., Shi, P., Liu, T. and Wang, C. (2013) Low concentrations of metformin selectively inhibit CD133(+) cell proliferation in pancreatic cancer and have anticancer action. PLoS ONE 8, e63969.

Green, A. S., Chapuis, N., Lacombe, C., Mayeux, P., Bouscary, D. and Tamburini, J. (2011) LKB1/AMPK/mTOR signaling pathway in hematological malignancies: from metabolism to cancer cell biology. Cell Cycle 10, 2115-2120.

Hardie, D. G. (2011) AMP-activated protein kinase: an energy sensor that regulates all aspects of cell function. Genes Dev. 25, 18951908.

Herrero-Martin, G., Hoyer-Hansen, M., Garcia-Garcia, C., Fumarola, C., Farkas, T., Lopez-Rivas, A. and Jaattela, M. (2009) TAK1 activates AMPK-dependent cytoprotective autophagy in TRAIL-treated epithelial cells. EMBO J. 28, 677-685.

Kang, G. J., Park, M. K., Byun, H. J., Kim, H. J., Kim, E. J., Yu, L., Kim, B., Shim, J. G., Lee, H. and Lee, C. H. (2020) SARNP, a participant in mRNA splicing and export, negatively regulates E-cadherin expression via interaction with pinin. J. Cell. Physiol. 235, 1543-1555.

Kang, S., Dong, S. M., Kim, B. R., Park, M. S., Trink, B., Byun, H. J. and Rho, S. B. (2012) Thioridazine induces apoptosis by targeting the PI3K/Akt/mTOR pathway in cervical and endometrial cancer cells. Apoptosis 17, 989-997. 
Lee, B. B., Kim, Y., Kim, D., Cho, E. Y., Han, J., Kim, H. K., Shim, Y. M. and Kim, D. H. (2019) Metformin and tenovin-6 synergistically induces apoptosis through LKB1-independent SIRT1 downregulation in non-small cell lung cancer cells. J. Cell. Mol. Med. 23, 2872-2889.

Lei, Y., Yi, Y., Liu, Y., Liu, X., Keller, E. T., Qian, C. N., Zhang, J. and Lu, Y. (2017) Metformin targets multiple signaling pathways in cancer. Chin. J. Cancer 36, 17.

Lengyel, E., Litchfield, L. M., Mitra, A. K., Nieman, K. M., Mukherjee, A., Zhang, Y., Johnson, A., Bradaric, M., Lee, W. and Romero, I. L. (2015) Metformin inhibits ovarian cancer growth and increases sensitivity to paclitaxel in mouse models. Am. J. Obstet. Gynecol. 212, 479.e1-479.e10.

Li, J., Zhong, L., Wang, F. and Zhu, H. (2017) Dissecting the role of AMP-activated protein kinase in human diseases. Acta Pharm. Sin. B 7, 249-259.

Li, M., Li, X., Zhang, H. and Lu, Y. (2018) Molecular mechanisms of metformin for diabetes and cancer treatment. Front. Physiol. 9, 1039.

Liu, Y., Marks, K., Cowley, G. S., Carretero, J., Liu, Q., Nieland, T. J., Xu, C., Cohoon, T. J., Gao, P., Zhang, Y., Chen, Z., Altabef, A. B., Tchaicha, J. H., Wang, X., Choe, S., Driggers, E. M., Zhang, J., Bailey, S. T., Sharpless, N. E., Hayes, D. N., Patel, N. M., Janne, P. A., Bardeesy, N., Engelman, J. A., Manning, B. D., Shaw, R. J., Asara, J. M., Scully, R., Kimmelman, A., Byers, L. A., Gibbons, D. L., Wistuba, I. I., Heymach, J. V., Kwiatkowski, D. J., Kim, W. Y., Kung, A. L., Gray, N. S., Root, D. E., Cantley, L. C. and Wong, K. K. (2013) Metabolic and functional genomic studies identify deoxythymidylate kinase as a target in LKB1-mutant lung cancer. Cancer Discov. 3, 870-879.

Loubiere, C., Clavel, S., Gilleron, J., Harisseh, R., Fauconnier, J., BenSahra, I., Kaminski, L., Laurent, K., Herkenne, S., Lacas-Gervais, S., Ambrosetti, D., Alcor, D., Rocchi, S., Cormont, M., Michiels, J. F., Mari, B., Mazure, N. M., Scorrano, L., Lacampagne, A., Gharib, A., Tanti, J. F. and Bost, F. (2017) The energy disruptor metformin targets mitochondrial integrity via modification of calcium flux in cancer cells. Sci. Rep. 7, 5040.

Mallik, R. and Chowdhury, T. A. (2018) Metformin in cancer. Diabetes Res. Clin. Pract. 143, 409-419.

Nangia-Makker, P., Yu, Y., Vasudevan, A., Farhana, L., Rajendra, S. G., Levi, E. and Majumdar, A. P. (2014) Metformin: a potential therapeutic agent for recurrent colon cancer. PLoS ONE 9, e84369.

Qu, C., Zhang, W., Zheng, G., Zhang, Z., Yin, J. and He, Z. (2014) Metformin reverses multidrug resistance and epithelial-mesenchymal transition (EMT) via activating AMP-activated protein kinase (AMPK) in human breast cancer cells. Mol. Cell. Biochem. 386, 63-71.

Rho, S. B., Song, Y. J., Lim, M. C., Lee, S. H., Kim, B. R. and Park, S. Y. (2012) Programmed cell death 6 (PDCD6) inhibits angiogenesis through $\mathrm{PI} 3 \mathrm{~K} / \mathrm{mTOR} / \mathrm{p} 70 \mathrm{~S} 6 \mathrm{~K}$ pathway by interacting of VEGFR-2. Cell. Signal. 24, 131-139.

Sanli, T., Rashid, A., Liu, C., Harding, S., Bristow, R. G., Cutz, J. C., Singh, G., Wright, J. and Tsakiridis, T. (2010) lonizing radiation activates AMP-activated kinase (AMPK): a target for radiosensitization of human cancer cells. Int. J. Radiat. Oncol. Biol. Phys. 78, 221-229.

Shaw, R. J., Kosmatka, M., Bardeesy, N., Hurley, R. L., Witters, L. A., DePinho, R. A. and Cantley, L. C. (2004) The tumor suppressor LKB1 kinase directly activates AMP-activated kinase and regulates apoptosis in response to energy stress. Proc. Natl. Acad. Sci. U.S.A. 101, 3329-3335.

Shaw, R. J., Lamia, K. A., Vasquez, D., Koo, S. H., Bardeesy, N., Depinho, R. A., Montminy, M. and Cantley, L. C. (2005) The kinase
LKB1 mediates glucose homeostasis in liver and therapeutic effects of metformin. Science 310, 1642-1646.

Steinberg, G. R. and Kemp, B. E. (2009) AMPK in health and disease. Physiol. Rev. 89, 1025-1078.

Suh, D. H., Lee, S., Park, H. S. and Park, N. H. (2020) Medroxyprogesterone reverses tolerable dose metformin-induced inhibition of invasion via matrix metallopeptidase-9 and transforming growth factor- $\beta 1$ in KLE endometrial cancer cells. J. Clin. Med. 9, 3585.

Sun, Y., Connors, K. E. and Yang, D. Q. (2007) AICAR induces phosphorylation of AMPK in an ATM-dependent, LKB1-independent manner. Mol. Cell. Biochem. 306, 239-245.

Towler, M. C. and Hardie, D. G. (2007) AMP-activated protein kinase in metabolic control and insulin signaling. Circ. Res. 100, 328-341.

Triggle, C. R. and Ding, H. (2017) Metformin is not just an antihyperglycaemic drug but also has protective effects on the vascular endothelium. Acta Physiol. 219, 138-151.

Uehara, T., Mitsuhashi, A., Tsuruoka, N. and Shozu, M. (2015) Metformin potentiates the anticancer effects of cisplatin under normoxic conditions in vitro. Oncol. Rep. 33, 744-750.

Vial, G., Detaille, D. and Guigas, B. (2019) Role of mitochondria in the mechanism(s) of action of metformin. Front. Endocrinol. 10, 294.

Wang, W. and Guan, K. L. (2009) AMP-activated protein kinase and cancer. Acta Physiol. 196, 55-63.

Whang, Y. M., Park, S. I., Trenary, I. A., Egnatchik, R. A., Fessel, J. P., Kaufman, J. M., Carbone, D. P. and Young, J. D. (2016) LKB1 deficiency enhances sensitivity to energetic stress induced by erlotinib treatment in non-small-cell lung cancer (NSCLC) cells. Oncogene $35,856-866$.

Xiao, X., He, Q., Lu, C., Werle, K. D., Zhao, R. X., Chen, J., Davis, B. C., Cui, R., Liang, J. and Xu, Z. X. (2012) Metformin impairs the growth of liver kinase B1-intact cervical cancer cells. Gynecol. Oncol. 127, 249-255.

Yuan, H. X., Xiong, Y. and Guan, K. L. (2013) Nutrient sensing, metabolism, and cell growth control. Mol. Cell 49, 379-387.

Zakikhani, M., Dowling, R., Fantus, I. G., Sonenberg, N. and Pollak, M. (2006) Metformin is an AMP kinase-dependent growth inhibitor for breast cancer cells. Cancer Res. 66, 10269-10273.

Zhang, J. and Snyder, S. H. (1992) Nitric oxide stimulates auto-ADPribosylation of glyceraldehyde-3-phosphate dehydrogenase. Proc. Natl. Acad. Sci. U.S.A. 89, 9382-9385.

Zhang, J., Xu, H., Zhou, X., Li, Y., Liu, T., Yin, X. and Zhang, B. (2017) Role of metformin in inhibiting estrogen-induced proliferation and regulating ERalpha and ERbeta expression in human endometrial cancer cells. Oncol. Lett. 14, 4949-4956.

Zhang, Y., Storr, S. J., Johnson, K., Green, A. R., Rakha, E. A., Ellis, I. O., Morgan, D. A. and Martin, S. G. (2014) Involvement of metformin and AMPK in the radioresponse and prognosis of luminal versus basal-like breast cancer treated with radiotherapy. Oncotarget 5, 12936-12949.

Zhao, D., Long, X. D., Lu, T. F., Wang, T., Zhang, W. W., Liu, Y. X., Cui, X. L., Dai, H. J., Xue, F. and Xia, Q. (2015) Metformin decreases IL-22 secretion to suppress tumor growth in an orthotopic mouse model of hepatocellular carcinoma. Int. J. Cancer 136, 2556-2565.

Zhu, Z., Jiang, T., Suo, H., Xu, S., Zhang, C., Ying, G. and Yan, Z. (2021) Metformin potentiates the effects of anlotinib in NSCLC via AMPK/mTOR and ROS-mediated signaling pathways. Front. Pharmacol. 12, 712181.

Zi, F. M., He, J. S., Li, Y., Wu, C., Yang, L., Yang, Y., Wang, L. J., He, D. H., Zhao, Y., Wu, W. J., Zheng, G. F., Han, X. Y., Huang, H., Yi, Q. and Cai, Z. (2015) Metformin displays anti-myeloma activity and synergistic effect with dexamethasone in in vitro and in vivo xenograft models. Cancer Lett. 356, 443-453. 